\title{
El uso de una plataforma de directivas anticipadas y preferencias del cuidado en los EE.UU. aumentó durante la pandemia de COVID-19
}

\author{
Completion of advanced directives and care preferences increased during COVID-19 pandemic in the USA
}

\section{Comentado de:}

Auriemma CL, et al. JAMA Netw Open. 2020;3(7):e2015762. PMID: $32687585^{1}$

\section{Objetivo}

Cuantificar los cambios en el llenado de directivas anticipadas (DA) y la expresión de preferencias del cuidado durante la pandemia de COVID-19 mediante el monitoreo de usuarios de una plataforma de DA en línea entre enero de 2019 y abril de 2020.

\section{Diseño, lugar y población}

Estudio de cohorte prospectiva de usuarios de OurCareWishes.org, una herramienta gratuita diseñada para guiar a los pacientes y sus familias durante el planeamiento de los cuidados avanzados.

\section{Medición de resultados principales}

Se evaluaron las tasas mensuales de llenado de DA, el número de módulos por objetivos completados y las distribuciones de preferencias del cuidado, antes y durante la pandemia.

Se definieron dos periodos: a) pre-COVID-19: enero de 2019 a enero de 2020 (momento en que los EE.UU. declararon la emergencia de salud pública) y b) COVID-19, Febrero a Abril de 2020. No se realizaron esfuerzos especiales para la diseminación de la plataforma en este último periodo.

Se usaron modelos de regresión logística multivariados para calcular las probabilidades de completar los módulos, clasificando ciertas prioridades cercanas al fin de la vida como extremadamente importantes, y ciertos estados de salud percibidos como similares o peores a una muerte confortable. Todos los modelos fueron ajustados por edad, y los que evaluaban las elecciones de DA además incluyeron el estado de salud autorreportado.

\section{Resultados principales}

Con un total de 424 (en 12 meses) y 482 usuarios (en 3 meses) antes y durante la pandemia, respectivamente, el número de usuarios por mes aumentó 4,9 veces entre ambos periodos evaluados (ver Tabla 1).

Tabla 1. Distribución mensual de los usuarios de la plataforma OurCareWishes.org en los periodos pre-COVID-19 y COVID-19.

\begin{tabular}{|l|c|c|c|}
\hline Periodo evaluado & Usuarios totales, Mediana (PIC) & Nuevos usuarios, Mediana (PIC) & Usuarios recurrentes, Mediana (PIC) \\
\hline Pre-COVID-19 & 31 (28 a 34) & 26 (22 a 30) & 5 (3 a 6) \\
\hline COVID-19 & 154 (82 a 246) & 133 (71 a 207) & 21 (11 a 39) \\
\hline
\end{tabular}

Tabla 2. Proporción de terminación de los cinco módulos optativos de la plataforma OurCareWishes.org en los que se constataron modificaciones significativas al comparar los periodos antes (Pre-COVID-19) y durante (COVID-19) la pandemia.

\begin{tabular}{|l|c|c|c|}
\hline Módulos & Pre-COVID-19, N (\%) & COVID-19, N (\%) & P-valor \\
\hline Identificación de objetivos de cuidado & $110(25,9 \%)$ & $201(41,7 \%)$ & $<0,001$ \\
\hline Prioridades importantes del final de la vida & $101(23,8 \%)$ & $181(37,6 \%)$ & $<0,001$ \\
\hline Clasificación de los estados de salud & $106(25,0 \%)$ & $198(41,1 \%)$ & $<0,001$ \\
\hline Donación de órganos & $110(25,9 \%)$ & $186(38,6 \%)$ & $<0,001$ \\
\hline Deseos para los días finales & $54(12,7 \%)$ & $109(22,6 \%)$ & $<0,001$ \\
\hline
\end{tabular}

Los individuos que usaron el servicio en el periodo COVID-19 fueron más jóvenes en comparación con el periodo anterior (edad media, 49,3 [Intervalo de confianza del $95 \%, 47,8$ a 50,9] años vs. 51,8 [IC 95\%, 50,2 a 53,5] años; $P=0,03$ ), con mejor estado de salud autorreportado (Kruskal-Wallis $H, 4,06$; $P=0,04$ ). Las tasas de llenado aumentaron en cinco de nueve módulos opcionales (ver Tabla 2).

\section{Conclusiones}

Este estudio revela un aumento de 4,9 veces en la declaración de las DA mediante el uso de esta platafor- ma, así como un llenado más completo de los módulos para expresar las preferencias en el cuidado de las personas desde el inicio de la pandemia de COVID-19, en ausencia de esfuerzos contemporáneos para promoverla.

Fuente de financiamiento/Conflicto de interés de los autores: Centro de Investigación en Cuidados Paliativos y Enfermedad Avanzada (PAIR, por sus iniciales en inglés) y Centro para la Innovación en el Cuidado de la Salud, Universidad de Pennsylvania. CL Auriemma recibió una beca de entrenamiento (5T32HL098054-09) del Instituto Nacional del Corazón, los Pulmones y la Sangre de los EE.UU. No se reportaron conflictos de interés. 


\section{Comentario}

Las DA son la manifestación escrita de una persona capaz quien, consciente y libremente, expresa las opciones, preferencias o deseos que deben respetarse en la asistencia sanitaria por recibir cuando se produzcan circunstancias clínicas que le impidan comunicar personalmente su voluntad ${ }^{2}$.

En Argentina, desde 2009 contamos con una ley de derechos del paciente en su relación con los profesionales e instituciones de la salud que avala el uso de las $\mathrm{DA}^{3}$. Esta norma fue reglamentada en $2012^{4}$ y modificada por la ley $26.742^{5}$, a la que se llamó "Ley de muerte digna". Los cambios surgieron tras un caso mediático en nuestro país que no solamente produjo movimientos e inquietudes en el poder legislativo, sino también en muchos ciudadanos ${ }^{6}$. $Y$ éste, creemos, es uno de los puntos de encuentro entre el artículo y la experiencia del Hospital Italiano de Buenos Aires (HIBA). A pesar de que esta institución avala el uso de las DA desde 1994, los primeros documentos registrados datan del año 2010, en relación a la implementación y difusión de un Programa de Directivas Anticipadas en 2009. Sin embargo, no fue hasta el 2011, cuando los medios de comunicación tomaron el caso de una mujer en situación de fin de vida con dolor intratable, que el número de documentos firmados por año comenzó a aumentar de manera exponencial, para luego, a partir de 2013, volver a descender. Probablemente, el colapso sanitario producido por la pandemia en los EE.UU. fue un gran estímulo para muchas de las personas que usaron la herramienta señalada en este estudio. La experiencia del Comité de Bioética del HIBA indica que las consultas sobre las DA y los documentos firmados suelen aumentar cuando las personas se sensibilizan frente a la problemática de los cuidados del final de la vida por alguna situación familiar vivida o por algún caso de un paciente que adquiere difusión masiva en los medios periodísticos. Estas dos situaciones han resultado mucho más efectivas que los esfuerzos destinados a difundir el tema entre los profesionales de la salud.

Sin embargo, creemos primordial que los médicos de cabecera pierdan el miedo a hablar de la muerte con sus pacientes y puedan preguntar sobre estos temas, de manera abierta y cuidadosa. Una escucha activa y atenta y una formación adecuada sobre los derechos de los pacientes pueden favorecer enormemente la toma de decisiones de manera anticipada.

\section{Conclusiones de las comentadoras}

Las DA son una herramienta que permite adecuar los cuidados de salud de las personas a sus deseos y preferencias en circunstancias que les impiden expresarlos o comunicarlos en forma directa al equipo médico. La crisis del sistema sanitario a causa de la pandemia de COVID-19 parece haber sido un estímulo importante en los EE.UU. para que más personas declaren sus DA en ese contexto, mediante una plataforma en línea. Los profesionales de la salud debemos estar preparados para acompañar a los pacientes en la toma de decisiones anticipadas, en forma acorde a la legislación que ampara sus derechos a un cuidado humanizado, en el que se respeten sus valores y preferencias.

Juliana Camoirano [ Servicio de Medicina Familiar y Comunitaria, Hospital Italiano de Buenos Aires; Comité de Bioética, Hospital Italiano de Buenos Aires. juliana.camoirano@hospitalitaliano.org.ar ]

Valeria Vietto [ Servicio de Medicina Familiar y Comunitaria, Hospital Italiano de Buenos Aires; Departamento de Investigación, Instituto Universitario Hospital Italiano de Buenos Aires. valeria.vietto@hospitalitaliano.org.ar ]

Camoirano J, Vietto V El uso de una plataforma de directivas anticipadas y preferencias del cuidado en los EE.UU. aumentó durante la pandemia de COVID-19 . Evid Actual Pract Ambul. 2020;23(3):e002090. Comentado de: Auriemma CL, et al. Completion of Advance Directives and Documented Care Preferences During the Coronavirus Disease 2019 (COVID-19) Pandemic. JAMA Netw Open. 2020;3(7):e2015762. PMID: 32687585

\section{Referencias}

1. Auriemma CM, Halpern SD, Asch JM, et al. Completion of Advance Directives and Documented Care Preferences During the Coronavirus Disease 2019 (COVID-19) Pandemic. AMA Netw Open. 2020;3(7):e2015762. Available from: 0.1001/jamanetworkopen.2020.15762.

2. Pezzano L. Directivas anticipadas. Una expresión de planificación anticipada del cuidado médico. Rev Hosp Ital B Aires. $2006 ; 26(4): 158-162$. Available from: https://www.hospitalitaliano.org.ar/multimedia/archivos/noticias_attachs/47/documentos/10378_2006.4.158-.Bioetica.pdf.

3. Argentina. Ministerio de justicia y derechos humanos. Derechos del Paciente en su relación con los Profesionales e Instituciones de la Salud; 2009. Available from: http://servicios.infoleg.gob.ar/infoleglnternet/anexos/160000-164999/160432/norma.htm [Last access: 2020-08-26].

4. Argentina. Ministerio de justicia y derechos humanos. Decreto 1089/2012. ; 2012. Available from: http://servicios.infoleg.gob.ar/infoleglnternet/ anexos/195000-199999/199296/norma.htm [Last access: 2020-08-26].

5. Argentina. Ministerio de justicia y derechos humanos. Ley 26.742.; 2012. Available from: http://servicios.infoleg.gob.ar/infoleglnternet/anexos/ 195000-199999/197859/norma.htm [Last access: 2020-08-26].

6. Hernández V. En Argentina la muerte digna se convierte en un derecho; 2012. Available from: https://www.bbc.com/mundo/noticias/2012/05/120510 argentina_ley_muerte_digna_senado_vh [Last access: 2020-08-26] 